\title{
MULTI-TRACKING SYSTEM FOR VEHICLE USING GPS AND GSM
}

\author{
Yuvraj Kasture $^{1}$, Suraj Gandhi ${ }^{2}$, Shravan Gundawar ${ }^{3}$, Ajinkya Kulkarni ${ }^{4}$ \\ 1, 2, 3, ${ }^{4}$ Student, Computer Engineering, UCOER, Maharashatra, India
}

\begin{abstract}
Multi-tracking system is a real time tracking platform which uses integration of technologies such as GPS and GSM. The platform supports multiple tracking devices for variety of applications such as live vehicle tracking, personal tracking and also assets tracking. The GPS device installed in the vehicle continuously moves with the vehicle and will calculate the co-ordinates with other related information at each position and then transmit this information via GSM to the tracking server, thus storing it in the database; which further can be viewed on electronic map, i.e., Google Map via Internet providing up-to-date information. This proposed system also supports for real time control like, if owner sends an SMS, it automatically turns of the ignition of vehicle or other different purposes. The overall system will be implemented in Micro-soft .NET technology in which C\#.Net will be used for system components \& for web based ASP.Net will be used.
\end{abstract}

Keywords: GPS, GSM, SMS, Socket Listener, Tracking server.

\section{INTRODUCTION}

The proposed system is reliable which is helpful in tracking multiple objects in real time. The vehicle tracking device is classified as active and passive where passive devices store GPS data and later this device is removed and downloaded to a computer for evaluation whereas, active devices collect the data and transmit it in real time over GSM network to the data center for evaluation [3]. The multi-tracking system thus contains two major components, i.e. GPS and GSM which is integrated in device which allows us to track the vehicle and generate different alerts.

\subsection{GPS and GSM:-}

GPS (Global Positioning System) [6]: It is a US owned utility that provides users with positioning, navigation and timing services. This system basically consists of three segments: the space segment, the control segment and user segment. Its uses between 24 and 32 medium earth orbit satellite that transmit précised microwave signals which enables GPS receivers to determine their current location, time and velocity.

GSM (Global System for Mobile Communication, originally Group Special Mobile) [7]: GSM is the pan European Mobile Telephony Standard specified by European Telecommunication Standards Institute, i.e. ETSI and provides common standard, thus cellular subscribers can use their mobile telephones all over Europe.

In this way, the proposed system can be useful in various applications such as live tracking for Emergency Services, Transport Company or State Transport Buses, personal tracking such as cabs, schools, etc.

\section{EXISTING SYSTEM}

\subsection{Automotive Navigation System}

An automotive navigation system [8] is a satellite navigation system designed for use in automobiles. It typically uses a GPS navigation device to acquire position data to locate the user on a road in the unit's map database. Using the road database, the unit can give directions to other locations along roads also in its database. a gyroscope and an accelerometer can be used for greater reliability, as GPS signal loss and/or multipath can occur due to tunnels. Some sorts can be taken out of the car and used hand-held while walking. This system is good for the driver only not for the owner of the vehicle who is not near to / far away the vehicle, so the owner cannot keep a watch on the driver as well as the vehicle.

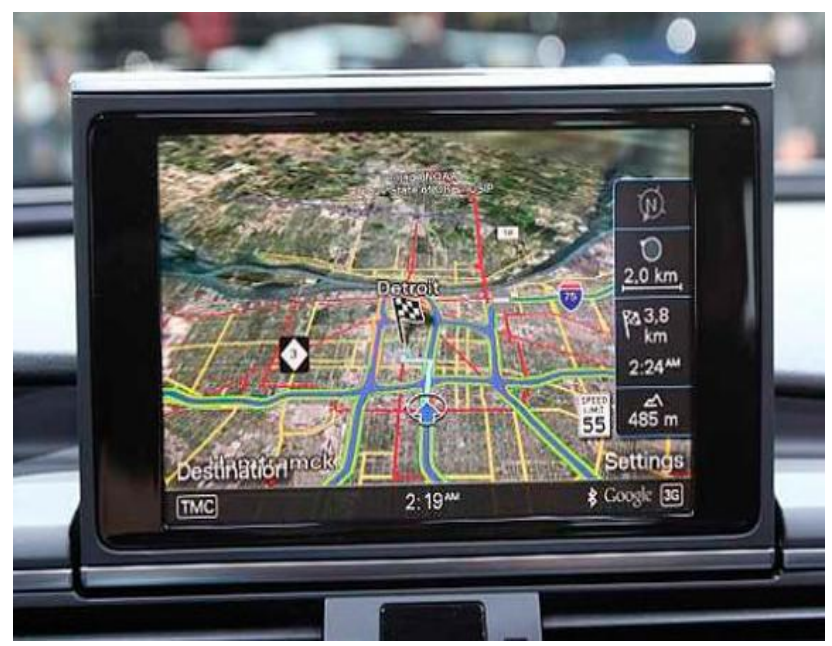

Fig -1: Automotive Navigation System 


\subsection{0pen GTS}

Open GTS is the abbreviation of "Open GPS Tracking System" [9] which is the first open source application specially designed to provide Web-based GPS tracking service for the vehicles. OpenGTS was designed to fill the needs of an entry-level fleet tracking system; it is also very highly configurable and scalable to larger enterprises as well. This system provides many features such as Web-based authentication Customizable web-page decorations, Operating system independent, Customizable mapping service, etc. and every feature has its own functionality and that is different than another.

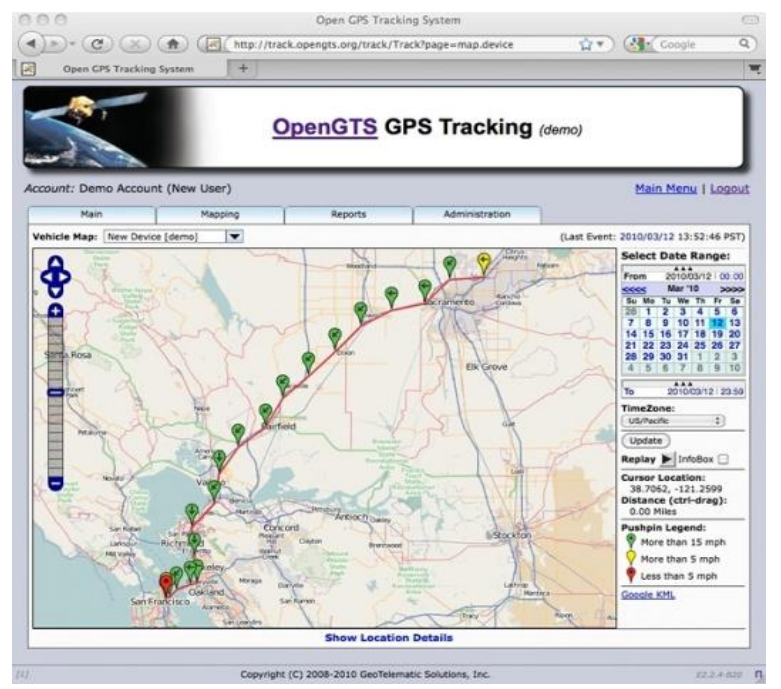

Fig -2: Open GTS

As mentioned above Customizable mapping service is one of the feature of OpenGTS which comes with support for OpenLayers/OpenStreetMap in addition to support for Google Maps, Microsoft Virtual Earth, and Mapstraction (which provides mapping support for MultiMap, Map24, MapQuest, and more). Within the OpenGTSframework, other mapping service providers can also easily be integrated.

\section{PROPOSED SYSTEM}

The major components of this system are as follows:
- AVL Device
- Tracking Server
- User Interface

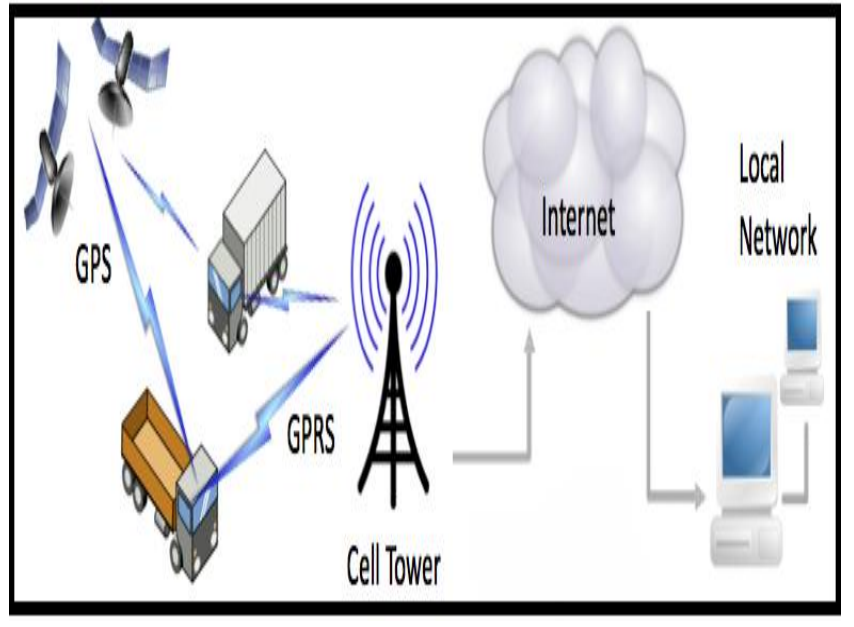

Fig -3: Physical working of the proposed system

The AVL (Advance Vehicle Locator) device which is placed in vehicle that accepts data from GPS satellites and stores it temporarily in the device. The device is installed with a SIM card which is useful for the purpose of communication with local network. Now, the data in the device is sent to the tracking server over GSM for further operation. This tracking server contains all data from AVL devices placed in the vehicles. Later on, as per the request of user the data from tracking server is displayed on user interface. That means, the user of the system interacts with the help of user interface provided. Also the registered user can interact with the system using cell phone, just by sending a text message. In this way, the overall system works thus generating reports and also generating alerts. GeoFence can be created on Google Maps which is provided, so whenever the device enters or leaves the geo fence, an alert will be sent to registered mobile number. There are web pages integrated with the Google Map and also for the purpose of viewing the vehicle on the GUI different API's are present.

\section{WORKING OF THE SYSTEM}

[1] We have already seen that the GPS satellite gives the position to the AVL device in the form of latitude, longitude as well as altitude. All this information is given to the tracking server which is then received to the socket listener on a particular port of the server. The data received from the device is in the hex format which cannot be read by humans. Using this raw data, a log file is prepared and further given to the parser [10] which parses the data according to the protocols of the device. After the parsing of the data, the data is converted into ASCII format which is readable by the human. The converted data is then stored then stored into the various fields of database for further use. As the complete data received from device is stored into database, we can use it whenever we want for live tracking, generating various reports, showing history, preparing KML [11] and other purposes also. 


\section{CONCLUSIONS}

With the help of multi tracking system we can track multiple vehicles using the AVL device. As system is having integration of GSM \& GPS we can use in it various applications. It is having wide scope of applications such as live tracking or personal tracking. You can determine your location, whether you are travelling locally or in a foreign land, having a GPS is truly an advantage and many more similar applications thus, this system can prove to be very helpful in future, providing more security.

\section{REFERNCES}

[1]. Sameer Darekar, Atul Chikane, Rutujit Diwate, Amol Deshmukh "Tracking System using GPS and GSM: Practical Approach", Issue 5, May-2012 1 ISSN 2229-5518 IJSER (C) 2012 http://www .ijser.org.

[2]. Kunal Maurya, Mandeep Singh, Neelu Jain "Real Time Vehicle Tracking System using GSM and GPS Technologyan Anti-theft Tracking System" ISSN- 2277-1956.

[3]. Patel Krishna Harshadbhai "Design of GPS and GSM Based Vehicle Location and Tracking System" IJSR 2319-7064 Volume 2 Issue 1, January 2013.

[4]. Abid khan, Ravi Mishra "GPS - GSM Based Tracking System” ISSN: 2231-5381.

[5]. Sathe Pooja "Vehicle Tracking System Using GPS" IJSR, Volume 2 Issue 9, September 2013.

[6]. GPS information; http://www.gsmarena.com/glossary.php3?term=gps

[7]. GSM information; http://ecee.colorado.edu/ ecen4242/gsm/index.htmg.

[8].

http://en.wikipedia.org/wiki/Automotive_navigation_system.

[9]. http://opengts.sourceforge.net/

[10]. FM4xxx Protocols and User Guides. For the study of protocols for the devices used for Tracking

[11]. http://en.wikipedia.org/wiki/Keyhole_Markup_Language.

\section{BIOGRAPHIES}

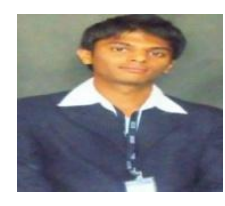

Yuvraj Kasture is currently pursuing bachelor's degree in Computer Engineering from Universal College of Engineering AND Research affiliated to Pune University, India. E-mail: yuvrajkasture99@gmail.com

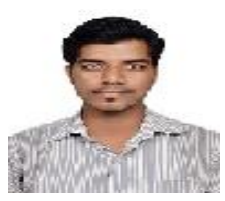

Suraj Gandhi is currently pursuing bachelor's degree in Computer Engineering from Universal College Of Engineering AND Research affiliated to Pune University, India. E-mail: suraj.gandhi@rocketmail.com

\section{KML;}

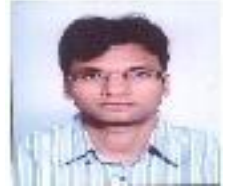

Shravan Gundawar is currently pursuing bachelor's degree in Computer Engineering from Universal College Of Engineering AND Research affiliated to Pune University,
$@$ yahoo.com. shravangundawar@yahoo.com.

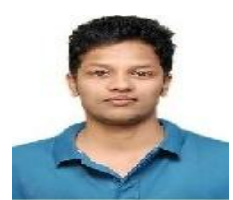

Ajinkya Kulkarni is currently pursuing bachelor's degree in Computer Engineering from Universal College Of Engineering AND Research affiliated to Pune University, ajukulkarni7@gmail.com
India. E-mail:

(1)

